\title{
Recent novel tumor gatekeepers and potential therapeutic approaches (2017)
}

\author{
Kashif Rafiq Zahid ${ }^{1 *}$, Hira Khan Zada ${ }^{2}$, Ghulam Mustafa Wassan ${ }^{2}$, Waseem \\ Hassan $^{3}$, Ke Liu ${ }^{1}$, Yanli Liu ${ }^{1}$, Jinlin Liu ${ }^{1}$, Haobin Zhao ${ }^{1}$ and Chao Qi ${ }^{1}$ \\ ${ }^{1}$ Hubei Key Laboratory of Genetic Regulation and Integrative Biology, College of Life Science, Central China Normal University, \\ Wuhan 430079, PR China, ${ }^{2}$ Department of Plant Breeding \& Genetics, Sindh Agriculture University, Tandojam, ${ }^{3}$ Department of \\ Soil and Environmental Sciences, Muhammad Nawaz Shareef University of Agriculture, Multan, Pakistan
}

*For correspondence: Email: qichao@mail.ccnu.edu.cn

Sent for review: 18 May 2017

Revised accepted: 20 September 2017

\begin{abstract}
Tumor remains a challenging task for oncology community. Drug resistance due to chemotherapy remain principal impediments toward potential therapeutic strategies. Development of novel anti-cancer drugs or new targeted strategies to conquer drug resistance is a key goal of cancer research. In this respect, novel tumor gatekeepers and innovative targeted strategies can be helpful in overcoming drug resistance as well as improve currently used targeted therapies. In this review, efforts have been made to present some of the latest knowledge about novel tumor gatekeepers and new therapeutic strategies to improve the efficacy of chemotherapy and give new hope to cancer patients to fight against cancer.
\end{abstract}

Keywords: Cancer, Potent inhibitors, Gatekeepers, Therapeutic approaches, Oncogenic pathways

Tropical Journal of Pharmaceutical Research is indexed by Science Citation Index (SciSearch), Scopus, International Pharmaceutical Abstract, Chemical Abstracts, Embase, Index Copernicus, EBSCO, African Index Medicus, JournalSeek, Journal Citation Reports/Science Edition, Directory of Open Access Journals (DOAJ), African Journal Online, Bioline International, Open-J-Gate and Pharmacy Abstracts

\section{INTRODUCTION}

Cancer is one of the main causes of human deaths worldwide. . Cancer is defined as a cluster of disorders characterized by unrestrained growth and multiplication of abnormal cells. Uncontrolled multiplication of abnormal cells leads to death. Approximately, cancer accounts one in four deaths. Life time cancer threat in women is (38\%) and men (42 $\%$ in the United States. American Cancer Society has reported that about 1,685,210 new cancer cases and nearly 595,690 deaths will be occurred in 2016 [1]. In 2017, approximately 600,920 peoples will be died from cancer [2].

Advances in cancer biology have led to development of novel anti-cancer drugs [3]. Efficacy of novel anti-cancer drugs is limited due to development of resistance as well as substantial toxicity. Recent studies have reported that resistance to chemotherapy is a main obstacle for efficient targeted cancer therapies $[4,5]$. To control cancers, there is urgent need to identify novel tumor gatekeepers and establishment of promising therapeutic approaches. In this review, we shed light on the recently identified new tumor gatekeepers and promising therapeutic approaches to conquer drug resistance.

\section{Role of novel tumor gatekeepers in cancer suppression}

Activation of the AKT oncogenic pathway through mutations in PIK3CA or PTEN loss is frequently occurred in many types of cancers; however, targeting of AKT pathway is a challenging task 
due to mechanism-based toxicities of the inhibitors which target inactive conformation of the AKT. A novel potent ATP-competitive inhibitor of the AKT (Ipatasertib), effectively targets active phosphorylated AKT [6].

More recent study has reported that this novel inhibitor confers vigorous and safe targeting of AKT in solid tumors. Authors have found that this inhibitor is tolerated; majority of the adverse events are gastrointestinal and grade 1-2 in severity. Exposure of novel inhibitor $\geq 200 \mathrm{mg}$ everyday in cancer patients is associated with TGI90, and results show that this selective novel inhibitor actively inhibits multiple targets such as mTOR, GSK3 $\beta$ and PRAS40 in paired ontreatment biopsies. Inhibition of the AKT signaling by with ipatasertib is correlated with significant control of cancer and tolerable safety profile in cancer patients. Targeting of phosphorylated AKT by using ATP-competitive inhibitor grants superior therapeutic window compared to allosteric inhibitors. Novel Ipatasertib inhibitor could be tumor gatekeeper in AKT-mediated human cancers [6].

Hepatocellular carcinoma is a severe human cancer. Fibroblast growth factor 19 is a ligand for the fibroblast growth factor 4 (FGF4) and plays critical role in hepatocellular carcinoma. Recently, it has been reported that novel potent inhibitor ASP5878 which successfully inhibits FGFR1, 2, 3 and 4, and also suppresses growth of FGF19-expressing hepatocellular carcinoma. This novel inhibitor suppresses the kinase activity of FGF4 with an $\mathrm{IC}_{50}$ of $3.5 \mathrm{nmol} / \mathrm{L}$. Moreover, this potent inhibitor suppresses FGF4 phosphorylation as well as its downstream signaling molecules and plus induces apoptosis. Furthermore, oral administration of ASP5878 (3 $\mathrm{mg} / \mathrm{kg}$ ) induces maintained tumor regression in the subcutaneous xenograft mouse model using Hep3B2.1-7, whereas ASP5878 induces complete tumor regression in $\mathrm{HuH}-7$, an orthotopic xenograft mouse model. These outcomes demonstrate that ASP5878 is a gatekeeper of hepatocellular carcinoma with tumors expressing FGF19 [7].

Polycomb repressive complex 2 (PRC2) regulates epigenetic states essential for homeostasis and development. This complex causes H3K4 trimethylation, consequently leading gene silencing and is deregulated in human cancers [8]. PRC2 is comprised of 3 core subunits, SUZ12, EED and EZH2, and controls transcriptional regulation. EZH2 is associated with the histone H3K3 methylation, and activity of $\mathrm{EZH} 2$ is increased after EED binding to the trimethylated H3K27.
More recent study has identified a selective and potent inhibitor EED226 which inhibits PRC2 by directly binding to EED H3K27me3 binding pocket [9]. This potent inhibitor induces a conformational change following binding of EED, and leads to loss PRC2 activity. Activity of this novel inhibitor is similar to SAM-competitive inhibitors in suppressing H3K27 methylation of the PRC2 target genes and inducing regression of the lymphoma xenograft tumors. Captivatingly, potent EED226 efficiently inhibits PRC2 containing a mutant EZH2 protein which cannot be inhibited by using SAM-competitive inhibitors. These novel findings show that a novel inhibitor EED226 successfully inhibits the activity of PRC2 through an allosteric mechanism and could be an authoritative gatekeeper of PRC2-dependent human cancers [9].

Moreover, another novel potent inhibitor A-395 has been reported which inhibits PRC2 complex activity via inhibiting EED protein-protein interaction. The results reveal that this novel inhibitor binds to the EED in the binding pocket of H3K27 trimethylation, thereby stopping allosteric activation of PRC2 catalytic activity. Activity of this potent inhibitor is similar to PRC2 enzymatic inhibitors both in vivo and in vitro; however, this potent inhibitor maintains its potent activity in response to those cell lines which show resistance against catalytic inhibitors. Thus, potent inhibitor A-395 represents a first-in-class antagonist of the PRC2 protein-protein interactions for use as a chemical probe for the investigation of novel functions of EEDcontaining protein complexes [8].

Protein lysine methyltransferases (PKMTs) are regulators of various physiological processes including transcription and genomic integrity maintenance. Genetic evidence shows that PKMTs (SUV42OH1 and SUV42OH2) promote non-homologous end-joining (NHEJ)-directed DNA repair via catalyzing di-and tri-methylation of lysine 20 on histone 4 [10]. Recently, Scientists have identified a selective and potent inhibitor A-196 which inhibits both the SUV420H1 and SUV420H2. Results show that A-196 is substrate-competitive inhibitor of SUV420H1 and SUV420H2 enzymes. This potent inhibitor induces a global reduction of H4K20me2 as well as H4K20me3 and plus a concomitant amplification of H4K20me1 in cells. This inhibitor inhibits foci formation of 53BP1 following ionizing radiation and decreases NHEJ-mediated DNA defects repair but does not affects homologydirected DNA repair. These novel outcomes show the significance of enzymatic activity of SUV4-20 in DNA repair H4K20 methylation. This potent inhibitor represents also a first-in-class 
SUV4-20 chemical probe in the investigation of importance of histonemethyltransferase in the genomic integrity [10].

Colden et al (2017) have reported that miRNA466 suppresses tumor growth and bone metastasis in the prostate tumor through direct regulation of RUNX2 osteogenic transcriptional factor. Results show that miR-466 is underexpressed in prostate tumor as compared to normal tissues. miR-466 reconstitution in metastatic prostate cancer cell lines impairs their oncogenic role including cell proliferation and invasion, and also induces apoptosis and cell cycle arrest as compared to control miRNA. On the contrary, miR-466 attenuation induces tumorigenic features in normal prostate cells. Moreover, miR-466 inhibits growth of prostate cancer and metastasis by targeting RUNX2 transcriptional factor. mir-466 overexpression leads to downregulation of integrated network of RUNX2 target genes such as osteocalcin, osteopontin, MMP11, ANGPTs including FAK, pAkt, Fyn and vimentin that are associated with invasion, migration, angiogenesis, metastasis and EMT. Furthermore, miR-466 suppresses growth of primary orthotopic tumor and spontaneous metastasis to bone.

Kaplan-Meir and receiver operating curve analyses indicate that expression of miR-466 is discriminated between malignant and normal prostate tissues; and predicts biochemical relapse. These findings establish that miR-466mediated attenuation of transcriptional factor RUNX2 as a new therapeutic tool for regulating growth of prostate cancer, especially metastasis to bone. For the first time, anti-bone function and clinical importance of miR-466 has been documented in the prostate cancer [11].

Cancer stem cells (CSCs) contribute to tumor recurrences and chemoresistance [12]. Recently, it has been reported that enforced miR-141 expression in CD44t and bulk $\mathrm{PCa}$ cells suppresses properties of CSCs such as invasion, sphere formation and holoclone, and inhibits tumor regeneration and metastasis. The expression of miR-141 enforces a powerful epithelial phenotype with an incomplete mesenchymal phenotype loss. Whole-genome RNA sequencing has uncovered new miR-141regulated molecular targets in in prostate cancer (PCa) cells including Rho GTPas family members such as ARPC5, CDC42, RAC1 and CDC42EP3 and stem cell molecules EZH2 and CD44, these are direct targets of miR-141. These novel findings demonstrate that miR-141 hampers tumor growth and metastasis via employing multiple mechanisms [13].
Latest study has presented a new presented a new chemoresistant prostate cancer patientderived xenograft (PDX) model in the NOD/SCID mice and discovered CD54 as a key gene among the highest enriched gene expression profiles in the prostate cancer exposed to chronic cisplatin admistration. Moreover, it has been observed that CD54 plays key role in tumorigenesis as well as self-renewal of prostate cancer stem cells. Knockdown of CD54 significantly decreases prostate cancer tumorigenesis both in vivo and in vitro and greatly extends survival time of tumorbearing mice in the prostate cancer xenograft model. Furthermore, it has been identified that p38-Notch1 axis is a major downstream signaling pathway in the CD54-mediated regulation of cancer stem cells in prostate tumors. These outcomes suggest that CD54 can be used as a prostate cancer stem cell marker and will provide a novel potential therapeutic target through CD54-Notch1 signaling in prostate cancer [12].

Lung cancer is a most common cancer and leading cause of deaths worldwide. Targeted therapies with tyrosine kinase inhibitors (TKIs) have been designed for the epidermal growth factor receptor (EGFR) positive patients in the adenocarcinoma or mixed adenosquamus lung cancer patients. However, novel chemotherapeutic agents have been developed for EGFR, at same time new mutations have also been found following TKI administration. Currently, new mutation T790 has been identified and is highly prevalent in the patients already treated with a tyrosine kinase inhibitor [14]. Osimertinib is a third generation, oral, pyrimidinebased, irreversible EGFR TKI which have got endorsement from FDA in 2015 to treat cancer patients with metastatic, EGFR T790M mutationpositive NSCLC resistant to EGFR TKI therapy. Osimertinib has been also approved for cancer patients with EGFR T790M mutation-positive NSCLC resistant to EGFR TKI therapy in European Union, Japan and United Kingdom. These findings show that Osimertinib acts as a novel gatekeeper for cancer patients with EGFR T790M mutation-positive NSCLC [15].

Molecular mechanism underlying cross-talk tumor-associated macrophages and tumor cells which induce metastasis in lung cancer is poorly studied. The scavenger receptor A1 (SR-A1) is primarily expressed in macrophages and is linked with lung cancer. Scientists have utilized transcriptomics, population genetics and functional analyses to explore how SR-A1 is engaged in lung tumorigenesis and its prognosis. They have investigated the SR-A1 genetic variants for feasible involvement in the survival of advanced-stage NSCLC patients in Harvard 
Lung Cancer Study cohort. Results show that 2 SNPs (rs1484751, rs17484273) in SR-A1 are linked with poor survival in this cohort. Significant SR-A1 downregulation in the lung tumor tissues has been observed. The involvement of SR-A1 with prognosis has been validated in animal models in the context of metastasis of lung cancer. Macrophages obtained from mice genetically deficient for the SR-A1 exhibited faster metastasis in lung cancer model [16] (Figure 1).

On the other hand, enhanced tumor cell seeding, invasion, migration and accumulation of macrophage in lung cancer tissue has been observed in SR-A1 deficient mice. Moreover, deletion of SR-A1 upregulates serum A1 (SAA1) in the macrophages through MAPK/lk-B/NF-kB signaling. SAA1 promotes macrophage migration and tumor cell invasion in vivo, but these effects are blocked by anti-SAA1 antibody administration. Collectively, these novel findings explain how SR-A1 inhibits metastasis of lung cancer via downregulating production of SAA1 in tumor associated macrophages [16].

\section{New strategies for drugging undrugable drug resistance}

Efficacy of tyrosine kinase inhibitors is satisfactory in the gastrointestinal stromal tumors (GISTs), but patients have received transitory benefits due to development of resistance to these inhibitors. Immunotherapy, especially blockade of ligand programmed death ligand 1 (PD-L1) or inhibitory receptor programmed death 1 (PD-1) has demonstrated well effectiveness in many types of cancers. Functional effects of PDL1 or PD-1 blockade remain elusive [17].

Scientists have identified that PD-L1/PD-1 blockade increases the activity of T-cell and imattinib anti-cancer efficacy in GISTs. Authors have found that T-cell immunoglobulin mucin-3, inhibitory receptors PD-1 and lymphocyte activation gene 3 are upregulated on the tumorinfiltrating T cells as compared to T cells from the matched blood. Meanwhile, variability has been found in intratumoral PD-L1 expression. Treatment with inhibitor imatinib abrogates IFNymediated PD-L1 upregulation through inhibition of STAT1 in GIST cell lines. In Kit ${ }^{7558 \Delta /+}$ mice, imatinib mediates downregulation of IFN-related genes and decreases expression of PD-L1 on the tumor cells.

Blockade of both PD-L1 and PD-1 did not show any efficacy alone in vivo but increase imatinib anti-tumor effects via enhancing the function of T-cell effector in the existence of IDO and KIT inhibition. PD-L1/PD-1 blockade is a well therapeutic approach to improve the efficacy of GISTs targeted therapy [17].

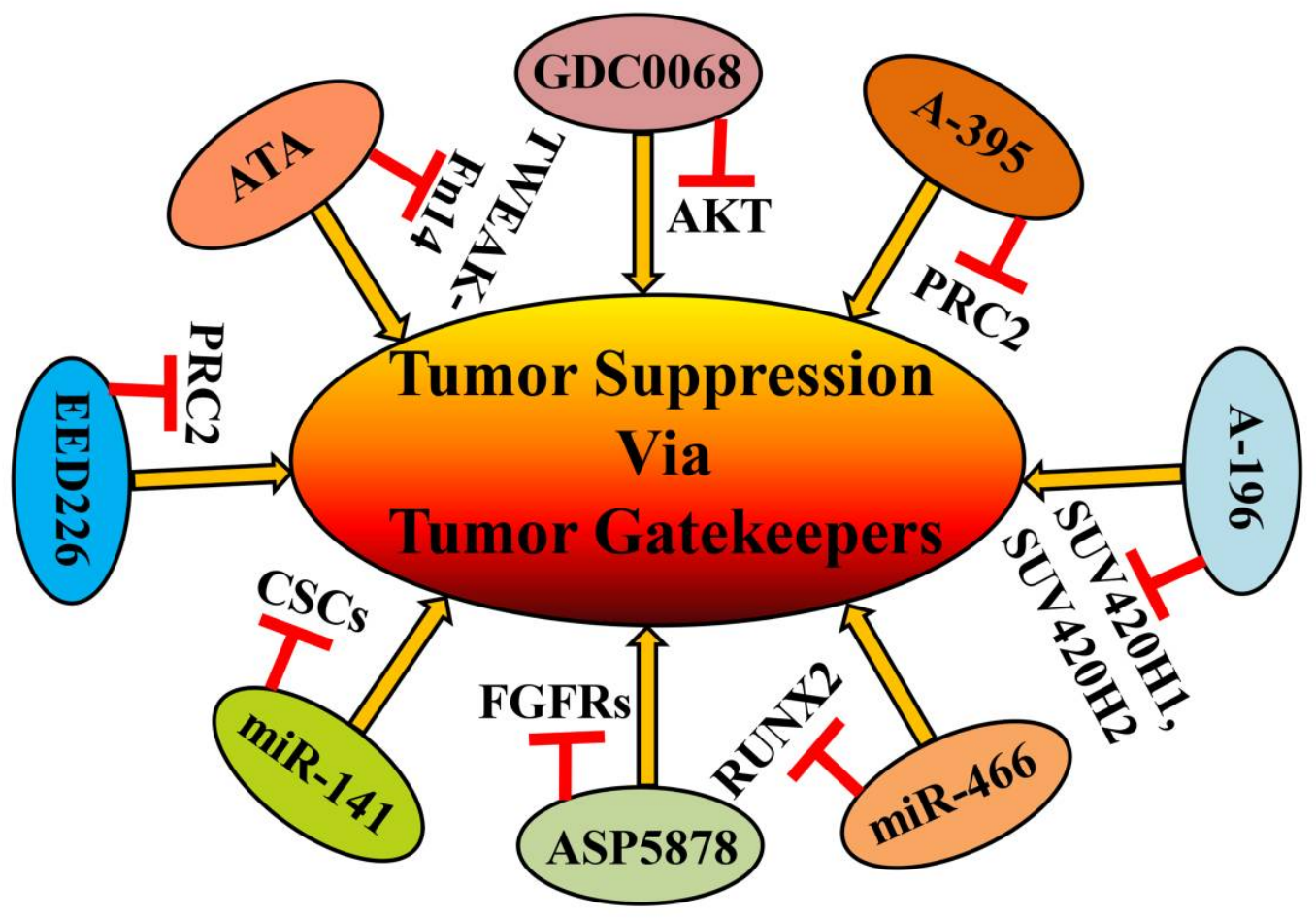

Figure 1: Schematic representation of tumor gatekeepers. Tumor gatekeepers effectively inhibits the various tumor drivers such as AKT, PRC2, SUV420H1/H2, RUNX2, FGFRs, CSCs, and TWEAK-Fn14-NF-kB dependent signaling 
Latest study has reported combination of adoptive cell therapy with vemurafenib in cancer patients with metastatic melanoma [18]. It has been observed that treatment is highly tolerated and shows safety profile same like TIL or vermurafenib alone. Among 11 cancer patients, 7 patients have showed goal of clinical response while 2 cancer patients have showed complete response for three years. Importantly, one response has been gone for about 4 years. Viability and proliferation of the peripheral blood $T$ cells and infusion bag TILS are suppressed in vitro with vermurafenib treatment under maximum vermurafenib serum concentration. TCRB repertoire (clone types, numbers, clonality, and frequency) and frequency) are not changed between, before and after vermurafenib lesions. Recognition of the autologous tumor via T cells is same between TILS is same between TILS grown from before and after vermurafenib metastases. Collectively, these findings show that coadminstration of TILS and vermurafenib is a feasible and safe granted target clinical response in clinical trial [18].

Growing evidence have shown that critical driver genomic events influence response to targeted agents in colorectal cancer, which have been designed under 'one gene, one drug' archetype of the precision medicine. However, recent information about cancer genome complexity, patterns of tumor evolution under pressure of anti-cancer drugs as well as pharmacodynamic effects of the target suppression support change from a one gene, one drug strategy to a 'multigene, multi-drug' approach during building therapeutic decisions. In colorectal cancer, transcriptomic subtypes characterization, encircling tumor, immune components and stromal have exposed dependencies of convergent pathway which mandate a 'multimolecular' viewpoint for designing targeted therapy to prevent this disease [19].

Therapy decision affects genetic landscape of the relapsed diseases. Scientists have identified that about $21 \%$ relapsed patients treated with $\mathrm{AL}$ have acquired CYP19A1 (encoding aromatase) amplification (CYP19A1 amp). Furthermore, relapsed patients have acquired various mutations targeting major breast cancer-linked genes such as CYP19A1 and ESR1. Particularly, emergence of CYP19A $1{ }^{\text {amp }}$ cells has been found in vitro in Al-resistant models. Amplification of CYP19A1 increases activity of aromatase as well as binding of estrogen-independent $E R \alpha$ to target genes, as result CYP19A $1^{\text {amp }}$ cells revealed reduced sensitivity to $\mathrm{Al}$ treatment. These results show that $\mathrm{Al}$ treatment itself chooses for acquired CYP19A $1^{\text {amp }}$ and enhances local autocrine estrogen signaling in the Alresistant metastatic patients [20].

Inhibition of SETD8 has been proposed a novel therapeutic approach for activation of p53 in the high-risk neuroblastoma. Given significance to scarcity of the druggable mutation in the high-risk neuroblastoma (NB), authors have undertaken chemical screens and epigenetic siRNA to explore epigenetic regulators important for blocking differentiation in high-risk neuroblastoma. High-content Opera imaging revealed that loss of 53 gene expression reduces cell proliferation in NB and 16 also induces differentiation. From these, SETD8, $\mathrm{H} 4^{\mathrm{K} 20 \mathrm{me} 1}$ methyltransferase has been identified as a drugagable NB target through secondary chemical screen.

Functional studies demonstrated that ablation of SETD8 rescued pro-apoptotic and cell cycle arrest p53 functions via reducing $\mathrm{p}^{\mathrm{K}}{ }^{\mathrm{K} 382 \mathrm{me} 1}$, promoting p53 canonical pathway activation. In the pre-clinical xenograft neuroblastoma models, pharmacological and genetic inhibition of SETD8 confers a remarkable survival benefit, providing convincing evidence that targeting of SETD8 is a novel therapeutic approach in neuroblastoma [21].

Stunning successes of the immunotherapy have revolutionized oncology field, and emerged as promising approach for solid tumors. Immunotherapies possess different action mechanisms and include CSF-1R antibodies, monoclonal antibodies, check point inhibitors, such as PD1/PD-L1 or CTLA-4, and oncolytic viruses [22]. Moreover, recent evidences show that immunotherapy combined with radiation therapy is more effective than monotherapy alone. Preclinical studies reveal that expression of PD-L1 is increased in tumor cells following radiation therapy, resulting synergistically increased antitumor effect of PD-L1 blockade and irradiation. In clinical setting, those cancer patients which received radiation therapy before anti-PD-1 treatment showed remarkably better prognosis than those who didn't receive radiation therapy [23].

A recent study has shown that destabilized tumor vasculature causes poor drug delivery, harmful tumor environment, hypoxia and even metastasis [24]. Scientists have done a side-by-side ABTAA (Ang2-binding and Tie2-activating antibody) comparison and ABA (Ang2-blocking antibody) in mice with orthotopically implanted glioma, with unprompted mammary cancer and 
subcutaneously implanted Lewis lung carcinoma. They have found that Tie2 activation induces tumor vascular normalization, promoting increased blood perfusion and delivery of chemotherapeutic drugs, noticeably reduces lactate acidosis, and decreases growth of tumor and metastasis. Furthermore, ABTAA positively alters profile of immune cell within tumors. These results demonstrate that simultaneous inhibition of Ang2 and activation of Tie2 shape a power full therapeutic window to build a sound tumor environment and successful chemotherapeutic agents delivery into tumors [24].

Genomic landscapes of cancers have been explored through The Cancer Genome Atlas. Similarly, immunotherapy has also transformed human cancer treatments. Unfortunately, a wide range of cancer patients do not respond to immunotherapy, suggesting the urgent need to identify predictive markers and resistance mechanism an area of powerful cancer study [25]. Recent evidence shows that immunosuppressive action of the regulatory $T$ cells is a major mechanism which limits success of immunotherapies with checkpoint blockers [26]. It has been reported that genotypeimmunophenotype associations and predictors of response to the checkpoint blockade can be identified through pan-cancer immunogenic analyses. To better understand tumor-immune cell interation, recent studies has characterized cancer antigenomes and intratumoral immune landscapes from twenty solid tumors and generated The Cancer Immune Atlas [25].

The hypothesis of cancer immunoediting suggests a dual function of immune system: protecting host through eliminating cancer cells, and shaping developing cancer via editing cancer genomes. Therefore, to what extent immunoediting shapes cancer genome in human malignancies is matter of debate. Furthermore, impact of immunotherapy with checkpoint blockers on modulating immunoediting has been poorly studied. [27].

Results show that Tregs infiltration and checkpoint molecules activation are main tumor escape mechanisms. Markedly, impact of the neutral evolution on sculpting tumor outweighed immunoediting via $T$ cell independent as well as $\mathrm{T}$ cell dependent mechanisms in progressing tumors. Scientists have also illustrated that PD1/PD-L1 pathway targeting potentiates immunoediting and makes tumor more homogenous. Also, these novel outcomes show that neutral evolution is a main force which sculpts tumor during progression, and blockade of checkpoint efficiently enforces $T$ cell dependent immunoselective pressure in this model. These novel findings have significant implication for the basic research studies on resistance mechanisms to checkpoint blockade and for the clinical translation [27].

Recent study has reported that EIF4E activation via aurora kinase $A$ illustrates a new druggable axis in the everolimus resistant cancer cells. It has been found that enhanced protein levels of AURKA promotes EIF4E phosphorylation and cMYC upregulation. Overexpression of endogenous AURKA has been detected in everolimus-resistant UGC cell models. AURKA mediated EIF4E phosphorylation, cap-dependent translation, and enhanced c-MYC protein levels. Targeting of AURKA through genetic knockdown or selective inhibitor, alisertib, reverses these events, leading to a decrease cancer cell survival in intrinsic and acquired resistant cell models.

Molecular studies indicate that AURKA binds to and inactivates EIF4E negative regulator protein phosphates 2A (PP2A), leading to EIF4E activation and resistance to everolimus in an AKT-ERK1/2, and mTOR-independent manner. Results from tumor xenograft mouse models showed that everolimus-resistant cancer cells are sensitive to alisertib. These data show that AURKA is strongly involved in EIF4E activation and cap-dependent translation. Targeting of AURKA-EIF4E-C-MYC axis through alisertib is a new therapeutic approach that could be applicable for those cancer subgroups that show upregulation of AURKA and C-MYC and EIF4E activation or everolimus-resistant tumors [28].

Latest study has reported that cancer stem cell marker CD271 is strongly involved in DNA damage response and drug resistance of melanoma cells. Results show that CD271 expression controls those genes which are linked with drug response and DNA repair. It was found that 110 CD271-responsive genes are significantly expressed in melanoma metastasis, of which, RAD51AP1, TOP2A and NEK2 were potential drivers of melanoma metastasis. They have identified that CD71 is regulated by p53 and in turn is required for an accurate p53dependent response to DNA-damaging drugs. This study has provided insight in a CD271mediated signaling network connecting CD271 with drug response, DNA repair and metastasis. Targeting of CD271 will be potential therapeutic strategy for overcoming drug resistance [29].

Neuroendocrine prostate cancer (NEPC) is a severe malignancy, molecular mechanisms which control the occurrence of NEPC, particularly those which are the consequences of 


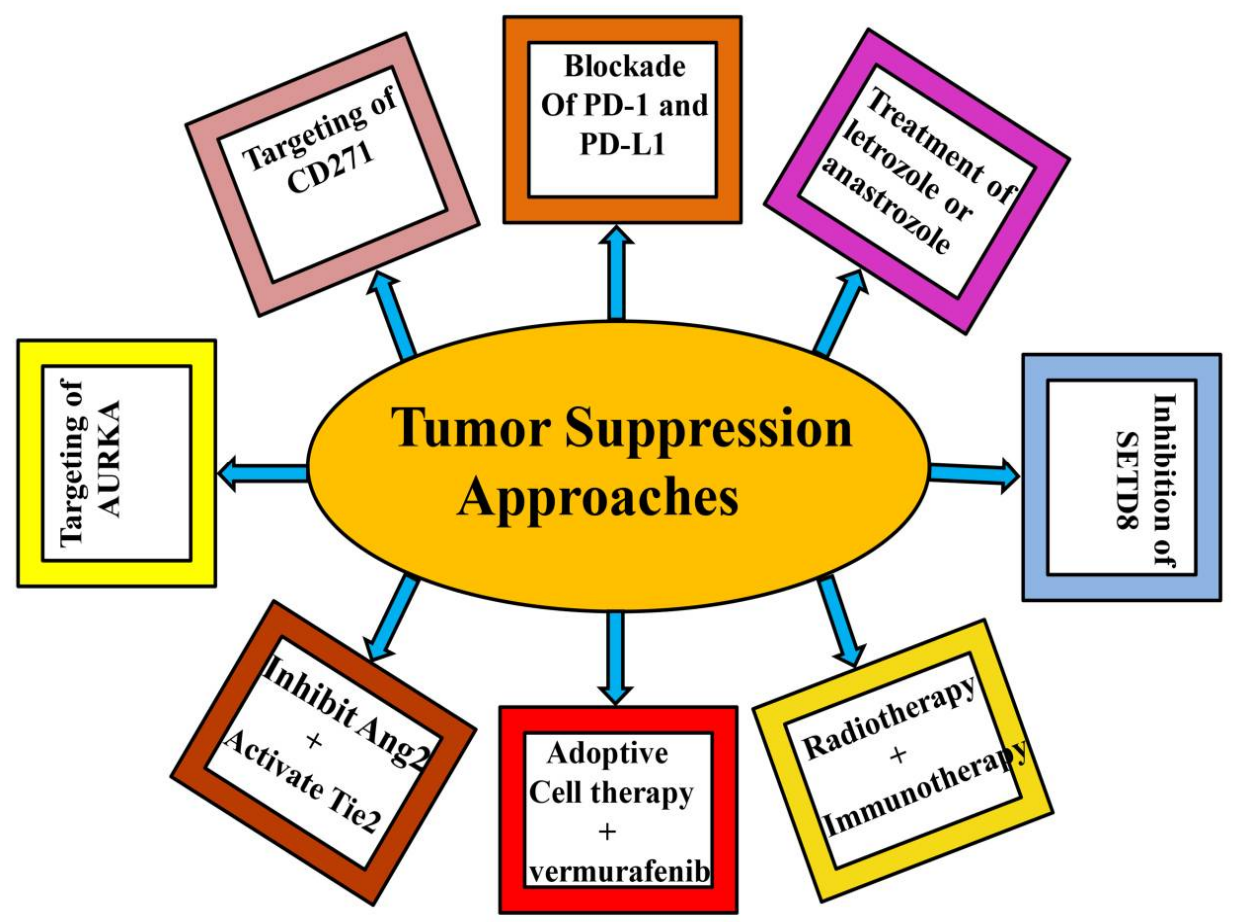

Figure 2: Schematic representation of potential therapeutic approaches. These novel approaches effectively targets various oncogenic agents and provide new therapeutic window to treat human cancer

treatment-induced inhibition of androgen receptor $(A R)$, remain elusive. More recently, latest study has discovered that control of neural BRN2 transcriptional factor (encoded by POU3F2) is a main driver of neuroendocrine prostate cancer and destructive tumor growth both in vivo and in vitro by using novel model of AR pathway inhibitor-resistant prostate cancer. Novel findings show that AR effectively suppresses the transcription of BRN2 which is needed for NEPC as well as BRN2-dependent regulation of NEPC marker SOX2. Underscoring its inverse correlation with the typical AR activity in the clinical samples, high expression of BRN2 was found in NETP cancers and was markedly enhanced in prostate cancer as compared to adenocarcinoma, particularly in the patients with low serum PSA. These findings have revealed a unique mechanism of AR-dependent control of NEPC, suggesting that BRN2 targeting is a novel approach to control neuroendocrin differentiation in the prostate cancer [30].

Squamous cell carcinoma in head and neck (HNSCC) is a common cancer but poorly explored, with adverse clinical results because of resistance to medication, recurrence and metastasis. Cancer stem cells have been found in the HNSCC, and expression of BMI1 is linked to these phenotypes, but therapeutic strategies to conquer chemotherapeutic resistance and overcome metastases have not been discovered yet. Latest study has reported that lineage tracing and genetic ablation which BMI1+ CSCs induce cervical lymph node metastasis and invasive growth in HNSCC mouse model. Primary HNSCC samples and this model hold extreme tumorigenic, invasive, and Cisplatinresistant $\mathrm{BMI} 1+\mathrm{CSCs}$, which possess enhanced AP-1 activity which mediates invasive growth and metastasis of the HNSCC. Inhibition of the AP-1 or BMl1 leads to sensitization of tumors to cisplatin-based chemical treatment, and it eradicate lymph node metastases via targeting bulk tumor and CSCs, indicating promising regimens to defeat resistance to medication and eliminate HNSCC metastasis [31] (Figure 2).

\section{CONCLUSION}

To win a battle against cancer, there is urgent need to identify novel tumor gatekeepers and establish new targeted therapeutic strategies. In this study, recent tumor gatekeepers and new therapeutic approaches have both been highlighted. It is hoped that this review will contribute to the efforts to overcome undruggable drug resistance and advance the design of new therapeutic windows for human treatment. 


\section{DECLARATIONS}

\section{Acknowledgement}

This study was supported by National Natural Science Foundation of China (nos. 31272645 and 31672284). Due to space limitation, several authors' original works could not be cited in this review.

\section{Conflict of Interest}

No conflict of interest associated with this work.

\section{Contribution of Authors}

The authors declare that this work was done by the authors named in this article and all liabilities pertaining to claims relating to the content of this article will be borne by them.

\section{Open Access}

This is an Open Access article that uses a funding model which does not charge readers or their institutions for access and distributed under the terms of the Creative Commons Attribution License (http://creativecommons.org/licenses/by/ 4.0) and the Budapest Open Access Initiative (http://www.budapestopenaccessinitiative.org/rea d), which permit unrestricted use, distribution, and reproduction in any medium, provided the original work is properly credited.

\section{REFERENCES}

1. Olaku OO, Taylor EA. Cancer in the Medically Underserved Population. Primary Care: Clinics in Office Practice 2017; 44(1): 87-97.

2. Siegel RL, Miller KD, Jemal A. Cancer statistics, 2017. CA Cancer J Clin 2017; 67(1): 7-30.

3. Lopez JS, Banerji U. Combine and conquer: challenges for targeted therapy combinations in early phase trials. Nat Rev Clin Oncol 2017; 14, 57-66.

4. Li R, Xie Y. Nanodrug delivery systems for targeting the endogenous tumor microenvironment and simultaneously overcoming multidrug resistance properties. J Control Rel 2017; 251, 49-67.

5. Tyszka-Czochara M, Konieczny P, Majka M. Caffeic Acid Expands Anti-Tumor Effect of Metformin in Human Metastatic Cervical Carcinoma HTB-34 Cells: Implications of AMPK Activation and Impairment of Fatty Acids De Novo Biosynthesis. Int J Molecul Sci 2017; 18(2): 462.

6. Saura $C$, Roda $D$, Roselló S, Oliveira M, Macarulla $T$, Pérez-Fidalgo JA, Morales-Barrera R, Sanchis-García JM, Musib L, Budha $N$ et al. A First-in-Human Phase I Study of the ATP-Competitive AKT Inhibitor Ipatasertib
Demonstrates Robust and Safe Targeting of AKT in Patients with Solid Tumors. Canc Discov 2017; 7(1): 102-113.

7. Futami $T$, Okada $H$, Kihara $R$, Kawase $T$, Nakayama $A$, Suzuki T, Kameda M, Shindoh N, Terasaka T, Hirano M et al ASP5878, a Novel Inhibitor of FGFR1, 2, 3, and 4, Inhibits the Growth of FGF19-Expressing Hepatocellular Carcinoma. Mol Canc Therapeut 2017; 16(1): 68-75.

8. He Y, Selvaraju S, Curtin ML, Jakob CG, Zhu H, Comess KM, Shaw B, The J, Lima-Fernandes E, Szewczyk MM et al. The EED protein-protein interaction inhibitor A-395 inactivates the PRC2 complex. Nat Chem Biol 2017.

9. Qi V, Zhao K, Gu J, Huang Y, Wang Y, Zhang H, Zhang $M$, Zhang J, Yu Z, Li L et al. An allosteric PRC2 inhibitor targeting the H3K27me3 binding pocket of EED. Nat Chem Biol. 2017.

10. Bromberg KD, Mitchell TR, Upadhyay AK, Jakob CG, Jhala MA, Comess KM, Lasko LM, Li C, Tuzon CT, Dai $Y$ et al. The SUV4-20 inhibitor A-196 verifies a role for epigenetics in genomic integrity. Nat Chem Biol 2017.

11. Colden M, Dar AA, Saini S, Dahiya PV, Shahryari V, Yamamura S, Tanaka Y, Stein G, Dahiya R, Majid S. MicroRNA-466 inhibits tumor growth and bone metastasis in prostate cancer by direct regulation of osteogenic transcription factor RUNX2. Cell Death \& Dis 2017; 8(1):e2572.

12. Li C, Liu S, Yan R, Han N, Wong KK, Li L.CD54NOTCH1 axis controls tumor initiation and cancer stem cell functions in human prostate cancer. Theranostics 2017; 7:67-80.

13. Liu C, Liu R, Zhang D, Deng Q, Liu B, Chao HP, Rycaj K, Takata $Y$, Lin K, Lu $Y$ et al. MicroRNA-141 suppresses prostate cancer stem cells and metastasis by targeting a cohort of pro-metastasis genes. Nat Communi 2017;8:14270

14. Zarogoulidis $P$, Gaga $M$, Huang $H$, Darwiche $K$, Rapti A, Hohenforst-Schmidt $W$. Tissue is the issue and tissue competition. Re-biopsy for mutation T790: where and why? Clin and Translat Medi 2017;6(1):6.

15. Soejima $K$, Yasuda $H$, Hirano $T$. Osimertinib for EGFR T790M mutation-positive non-small cell lung cancer. Expert Review of Clini Pharmacol 2017;10(1):31-8.

16. Zhang $Y$, Wei $Y$, Jiang $B$, Chen $L$, Bai $H$, Zhu $X$, Li X, Zhang $H$, Yang $Q$, Ma $J$ et al. Scavenger receptor $A 1$ prevents metastasis of non-small cell lung cancer via suppression of macrophage serum amyloid A1. Canc Res 2017.

17. Seifert AM, Zeng S, Zhang JQ, Kim TS, Cohen NA, Beckman MJ, Medina BD, Maltbaek JH, Loo JK, Crawely $\mathrm{MH}$ et al. (2017) PD-1/PD-L1 Blockade Enhances T-cell Activity and Antitumor Efficacy of Imatinib in Gastrointestinal Stromal Tumors. Clinic Canc Res 2017; 23.

18. Deniger DC, Kwong ML, Pasetto A, Dudley ME, Wunderlich JR, Langhan MM, Lee CC, Rosenberg SA. A Pilot Trial of the Combination of Vemurafenib with Adoptive Cell Therapy in Patients with Metastatic Melanoma. Clini Canc Res 2017; Clin Canc Res-0906.

Trop J Pharm Res, October 2017; 16(10): 2552 
19. Dienstmann R, Vermeulen L, Guinney J, Kopetz $S$, Tejpar S, Tabernero J. Consensus molecular subtypes and the evolution of precision medicine in colorectal cancer. Nat Reviews Canc 2017; 17:79-92.

20. Magnani L, Frigè G, Gadaleta RM, Corleone G, Fabris $S$, Kempe MH, Vershure PJ, Barozzi I, Vircillo V, Hong $S P$ et al. Acquired CYP19A1 amplification is an early specific mechanism of aromatase inhibitor resistance in ER [alpha] metastatic breast cancer. Nat Genet 2017.

21. Veschi V, Liu Z, Voss TC, Ozbun L, Gryder B, Yan C, Hu $Y$, Ma A, Jin J, Mazur SJ et al. Epigenetic siRNA and Chemical Screens Identify SETD8 Inhibition as a Therapeutic Strategy for p53 Activation in High-Risk Neuroblastoma. Canc Cell 2017; 31(1): 50-63.

22. Cousin S, Italiano A. Toxicity profiles of Immunotherapy. Pharmocology and Therapeutics 2017.

23. Takamori S, Toyokawa G, Takada K, Shoji F, Okamoto $T$, Maehara Y. Combination Therapy of Radiotherapy and Anti-PD-1/PD-L1 Treatment in Non-small Cell Lung Cancer, A Mini-Review. Clinic Lung Canc 2017.

24. Park JS, Kim IK, Han S, Park I, Kim C, Bae J, Oh SJ, Lee $S$, Kim JH, Woo DC et al. Normalization of Tumor Vessels by Tie2 Activation and Ang2 Inhibition Enhances Drug Delivery and Produces a Favorable Tumor Microenvironment. Canc Cell 2017; 31(1):157158.

25. Charoentong $P$, Finotello $F$, Angelova $M$, Mayer $C$, Efremova M, Rieder D, Hackl H, Trajanoski Z. Pancancer immunogenomic analyses reveal genotypeimmunophenotype relationships and predictors of response to checkpoint blockade. Cell Rep 2017; 18(1):248-262.

26. Finotello F, Trajanoski Z. New strategies for cancer immunotherapy: targeting regulatory $T$ cells. Genome Medi 2017; 9(1):10.

27. Trajanoski Z, Efremova $M$, Klepsch $V$, Charoentong $P$, Finotello $F$, Rieder D, Hackl H, Hermann-Kleiter N, Baier $G$, Krogsdam A. Targeting the PD-1/PD-L1 pathway potentiates immunoediting to counterbalance neutral evolution in a mouse model of colorectal cancer. bioRxiv 2017.

28. Katsha A, Wang L, Arras J, Omar OM, Ecsedy JA, Belkhiri A, El-Rifai W. Activation of EIF4E by Aurora kinase $A$ depicts a novel druggable axis in everolimus resistant cancer cells. Clinic Canc Res 2017.

29. Redmer T, Walz I, Klinger B, Khouja S, Welte Y, Schäfer $R$, Regenbrecht $C$. The role of the cancer stem cell marker CD271 in DNA damage response and drug resistance of melanoma cells. Oncogenesis 2017; 6:e291

30. Bishop JL, Thaper D, Vahid S, Davies A, Ketola K, Kuruma $H$, Jama R, Nip KM, Angeles A, Johnson $F$ et al. The Master Neural Transcription Factor BRN2 is an Androgen Receptor-Suppressed Driver of Neuroendocrine Differentiation in Prostate Cancer. Canc Discov 2017; 7(1): 54-71.

31. Chen D, Wu M, Li Y, Chang I, Yuan Q, Ekimyan-Salvo M, Deng $P, Y u B, Y u Y$, Dong $J$ et al. Targeting BMl1+ Cancer Stem Cells Overcomes Chemoresistance and Inhibits Metastases in Squamous Cell Carcinoma. Cell Stem Cell 2017. 